\title{
INTERNATIONAL JOURNAL OF HUMANOID ROBOTICS
}

\section{Author Index Volume 3 (2006)}

Aylett, R., see Hall, L.

Babič, J. and Lenarčič, J., Optimization of Biarticular Gastrocnemius Muscle in Humanoid Jumping Robot Simulation

Babković, K., see Potkonjak, V.

Bagheri, A., Najafi, F., Farrokhi, R., Moghaddam, R. Y. and Felezi, M. E., Design, Dynamic Modification, and Adaptive Control of a New Biped Walking Robot

Barrett, A., see Davies, B. L.

Becker, C., see Prendinger, H.

Blanchard, A. J., see Cañamero, L.

Borovac, B., see Potkonjak, V.

Borovac, B., see Vukobratović, M.

Bryson, J. J., see Tanguy, E.

Buisine, S., see Martin, J.-C.

Cañamero, L., Blanchard, A. J. and Nadel, J., Attachment Bonds for Human-Like Robots

Chan, C. F., see Phee, L.

Cheng, C., see Phee, L.

Chida, K., see Solis, J.

Cobb, J., see Davies, B. L.

Cosío, F. A., Padilla Castañeda, M. A. and Sevilla Martínez, P. R., Computer Assisted Prostate Surgery Training

Daerden, F., see Verrelst, B.

Dai, M., see Wu, Y.

Davies, B. L., Rodriguez, F., Jakopec, M., Harris, S. J., Barrett, A., Gomes, P., Henckel, J. and Cobb, J., The Acrobot ${ }^{\circledR}$ System for Robotic MIS Total Knee and UNI-Condylar Arthroplasty

Davies, B., see Zivanovic, A.

De La Rivière, J.-B. and Guitton, P., Human Motion Perception through Video Model-Based Tracking

Devillers, L., see Martin, J.-C.

Dibble, E., see Zivanovic, A.

Farrokhi, R., see Bagheri, A.

Felezi, M. E., see Bagheri, A.

Gomes, P., see Davies, B. L.

Guitton, P., see De La Rivière, J.-B.

Hall, L., Woods, S., Aylett, R. and Paiva, A., Using Theory of Mind Methods to Investigate Empathic Engagement with Synthetic Characters

Harada, K., Kajita, S., Kaneko, K. and Hirukawa, H., An Analytical Method for Real-Time Gait Planning forHumanoid Robots

Harris, S. J., see Davies, B. L.

3 (2006) 351

$\mathbf{2}(2006) 219$

1 (2006) 21

1 (2006) 105

4 (2006) 415

3 (2006) 371

3 (2006) 301

1 (2006) 21

2 (2006) 153

3 (2006) 293

3 (2006) 269

3 (2006) 301

4 (2006) 463

4 (2006) 463

2 (2006) 127

4 (2006) 415

4 (2006) 485

1 (2006) 67

2 (2006) 177

$4(2006) 415$

4 (2006) 429

3 (2006) 341

3 (2006) 269

4 (2006) 429

1 (2006) 105

1 (2006) 105

4 (2006) 415

3 (2006) 341

3 (2006) 351

1 (2006) 1

4 (2006) 415 
Henckel, J., see Davies, B. L.

$4(2006) 415$

Heylen, D., Head Gestures, Gaze and the Principles of Conversational Structure

Hirukawa, H., see Harada, K.

Ho, H., see Phee, L.

Ishizuka, M., see Prendinger, $\mathrm{H}$.

Ivancevic, V., Lie-Lagrangian Model for Realistic Human Biodynamics

Jakopec, M., see Davies, B. L.

Kajita, S., see Harada, K.

Kaneko, K., see Harada, K.

Lam, S. K., see Lim, C. S.

Lefeber, D., see Verrelst, B.

Lenarčič, J., see Babič, J.

Lim, C. S., Lam, S. K., Tian, H. and Srikanthan, T., Efficient Architectures for Segmentation of Endoscopic Images in Micro-Robotic Auto Navigation Systems

Ling, K. V., see Shao, F.

Ling, K. V., see Shao, W.

Low, S. C. and Phee, L., A Review of Master-Slave Robotic Systems for Surgery

Martin, J.-C., Niewiadomski, R., Devillers, L., Buisine, S. and Pelachaud, C., Multimodal Complex Emotions: Gesture Expressivity and Blended Facial Expressions

Moghaddam, R. Y., see Bagheri, A.

Muscato, G. and Spampinato, G., A Pneumatic Human Inspired Robotic Leg: Control Architecture and Kinematical Overview

Nadel, J., see Cañamero, L.

Najafi, F., see Bagheri, A.

Naudet, J., see Verrelst, B.

Ng, W. S., see Phee, L.

Ng, W. S., see Shao, F.

Ng, W. S., see Shao, W.

Niewiadomski, R., see Martin, J.-C.

Okita, S. Y. and Schwartz, D. L., Young Children's Understanding of Animacy and Entertainment Robots

Padilla Castañeda, M. A., see Cosío, F. A.

Paiva, A., see Hall, L.

Pelachaud, C., see Martin, J.-C.

Peters, C., A Perceptually-Based Theory of Mind for Agent Interaction Initiation

3 (2006) 241

$1(2006) 1$

$4(2006) 463$

$3(2006) 371$

$2(2006) 205$

$4(2006) 415$

$1(2006) 1$

$1(2006) 1$

$4(2006) 523$

$1(2006) 67$

$2(2006) 219$

4 (2006) 523

4 (2006) 439

4 (2006) 499

4 (2006) 547

3 (2006) 269

1 (2006) 105

1 (2006) 49

3 (2006) 301

1 (2006) 105

1 (2006) 67

4 (2006) 463

4 (2006) 439

4 (2006) 499

3 (2006) 269

3 (2006) 393

4 (2006) 485

3 (2006) 351

3 (2006) 269

3 (2006) 321

Phee, L., Yuen, J., Xiao, D., Chan, C. F., Ho, H., Thng, C. H., Tan, P. H., Cheng, C. and Ng, W. S., Ultrasound Guided Robotic Biopsy of the Prostate

$4(2006) 463$

$4(2006) 547$

$4(2006) 439$

Phee, L., see Shao, F.

Potkonjak, V., Vukobratović, M., Babković, K. and Borovac, B., General Model of Dynamics of Human and Humanoid Motion:

Feasibility, Potentials and Verification

1 (2006) 21

$2(2006) 153$

Potkonjak, V., see Vukobratović, M.

Prendinger, H., Becker, C. and Ishizuka, M., A Study in Users'

Physiological Response to an Empathic Interface Agent

$3(2006) 371$ 
Rodriguez, F., see Davies, B. L.

$4(2006) 415$

Schwartz, D. L., see Okita, S. Y.

3 (2006) 393

Sevilla Martínez, P. R., see Cosío, F. A.

Shao, F., Ling, K. V., Phee, L. and Ng, W. S. and Xiao, D., Efficient $3 D$ Prostate Surface Detection for Ultrasound Guided robotic Biopsy

Shao, W., Wu, R., Thng, C. H., Ling, K. V. and Ng, W. S., Integrating MRI and MRSI Information into Trus-Guided Robotic Prostate Biopsy Shen, J., see Wu, Y.

Solis, J., Chida, K., Suefuji, K. and Takanishi, A., The Development of the Anthropomorphic Flutist Robot at Waseda University

Spampinato, G., see Muscato, G.

Srikanthan, T., see Lim, C. S.

Suefuji, K., see Solis, J.

Takanishi, A., see Solis, J.

Tan, P. H., see Phee, L.

Tanguy, E., Willis, P. J. and Bryson, J. J., A Dynamic Emotion Representation Model within a Facial Animation System

Thng, C. H., see Phee, L.

Thng, C. H., see Shao, W.

$4(2006) 485$

$4(2006) 439$

$4(2006) 499$

$2(2006) 177$

$\mathbf{2}(2006) 127$

1 (2006) 49

$4(2006) 523$

2 (2006) 127

2 (2006) 127

4 (2006) 463

3 (2006) 293

$4(2006) 463$

$4(2006) 499$

$4(2006) 523$

1 (2006) 67

1 (2006) 67

1 (2006) 67

1 (2006) 67

Vermeulen, J., see Verrelst, B.

Verrelst, B., Vermeulen, J., Vanderborght, B., Van Ham, R., Naudet, J., Lefeber, D., Daerden, F. and Van Damme, M., Motion Generation and Control for the Pneumatic Biped "Lucy"

Vukobratović, M., Borovac, B. and Potkonjak, V., ZMP: A Review of Some Basic Misunderstandings

Vukobratović, M., see Potkonjak, V.

Willis, P. J., see Tanguy, E.

Woods, S., see Hall, L.

$\mathrm{Wu}, \mathrm{R}$., see Shao, W.

Wu, Y., Shen, J. and Dai, M., Self-Calibration: New Method and Comparisons

$1(2006) 67$

$\mathbf{2}(2006) 153$

1 (2006) 21

3 (2006) 293

$3(2006) 351$

$4(2006) 499$

$\mathbf{2}(2006) 177$

$4(2006) 463$

$4(2006) 439$

$4(2006) 463$

Xiao, D., see Shao, F.

Yuen, J., see Phee, L.

Zivanovic, A., Dibble, E. and Davies, B., A High Force Haptic System for Knee Arthroscopy Training

4 (2006) 429 